\title{
CRESTAL BONE LOSS USING DIFFERENT STUD ATTACHMENTS IN DISTAL EXTENSION CASES
}

\author{
Hesham I. AlmEldeen*
}

\begin{abstract}
The duality of support that characterizes distal extension removable partial dentures is often characterized by time-dependent adverse changes in both the abutment teeth and edentulous areas. Consequently, adjunctive implant has been proposed for mandibular class I and II removable partial denture designs. This current study evaluated the effect of locator attachment system and ball abutment with O-ring female metal housings in implant retained mandibular partial overdenture by measuring peri-implant crestal bone level. Twenty partially edentulous male patients exhibiting bilateral distal extension edentulous areas were selected. Two implants were installed in the first molar region following the two stage surgical technique. Patients were randomly divided into 2 equal groups; Group 1 locator attachments were used and Group 2 ball attachments were used. The mesial, distal, buccal and lingual marginal bone height around the implants was evaluated using cone beam CT. The measurements were carried out at insertion, six and twelve months post insertion. The results of this study showed a statistically significant increase in crestal bone height loss around the implants in both groups during the follow up period. In conclusion, ball -retained mandibular overdenture are associated with more marginal bone compared to locator abutments.
\end{abstract}

\section{INTRODUCTION}

Lack of support, stability and retention are the main problems of Kennedy class I cases restored with conventional RPD due to lack of distal abutment. There is lever action resulting from difference in resiliency between the mucoperiosteum and the periodontal ligament, this difference lead to destructive forces on abutments and supporting structures. A rotational movement usually occurs around the fulcrum of the terminal abutments when Functional occlusal load is applied on distal-extension removable partial denture. These phenomenons not only decrease the denture function and cause the patient's discomfort, but also traumatize the supporting tissues of the dentures..$^{(1,2)}$.

Lack of stability,minimal retention, periodontally compromised abutment teeth, and unaesthetic clasps are limitation of conventional, nonimplantsupported RPD. These limitations frequently are accompanied by discomfort, accelerated tooth loss,

* Lecturer, Prosthodontics Dept. Faculty of Oral and Dental Medicine Future University, Cairo, Egypt. 
and traumatic forces, as well as patient using the appliance routinely. Further, if a critical abutment tooth is lost, these appliances can be rendered useless $^{(3)}$.

Implantology made it possible for osseointegrated implants to be used in partially edentulous patients especially in Kennedy class I and II.) Implants incorporated into RPD provided support and retention through the use of different attachments. Placement of dental implants in conjunction with RPD may overcome most of the common problems with conventional $\operatorname{RPD}^{(4,5)}$.

Ball attachments provide for retention and patient satisfaction . The ball and socket attachments consist of a metal ball (male portion) which is screwed into the fixture, where the female part is incorporated in the fitting surface of the denture. It has been recommended that O-rings should be changed either annually, or biannually, depending on the number of implants used ${ }^{(6,7)}$.

Locator attachments can be a suitable alternative to ball attachments because of their low profile, when the inter-arch distance or the height of the denture is inadequate for placing ball attachment. Locator Attachment characterized by having different abutment heights ( 0 to $6 \mathrm{~mm}$ depending on the implant system) to allow for various soft tissue thicknesses, and also by having a low profile design, which means that the abutment does not protrude significantly above the marginal tissue height and also the male connector occupies less space within the denture. The lowest vertical height of the Locator Attachment (abutment and male) is $3.17 \mathrm{~mm}$ for externally hexed implants and only $2.5 \mathrm{~mm}$ for a non-hexed, internal connection implant. This is advantageous where there is limited interocclusal space $^{(8,9)}$. locator attachment system (Zest Anchors) is self-aligning and has dual retention (inner and outer retention) ${ }^{(10)}$.

\footnotetext{
* Bayer dental, Leverkusen, Germany
}

Monitoring marginal bone loss around implants is regarded by numerous authors as the most important criterion in determining the success of implants. This criterion is generally accepted as a reliable indicator of bone response to the surgical procedure and subsequent occlusal loading ${ }^{(11,12)}$.

This current study evaluated the effect of locator attachment system and ball abutment with $\mathrm{O}$ - ring female metal housings in implant-retained distal extension partial overdentures by measuring periimplant crestal bone level changes using Conebeam computed tomography.

\section{MATERIALS AND METHODS}

Twenty patients were selected from the outpatient clinic, Prosthodontic department, Faculty of Dentistry, Future University. The selected patients had the following criteria, all patients had Kennedy class I lower partially edentulous ridges with the first or second premolars were the last standing abutments. (Fig1) The remaining residual ridges had enough bone height, width and covered with firm mucosa, showing no signs of inflammation or ulceration.Patients age ranged from 55-65 years.

- Preliminary impressions were made for the upper and lower arches using irreversible hydrocolloid impression material ${ }^{*}$ in properly

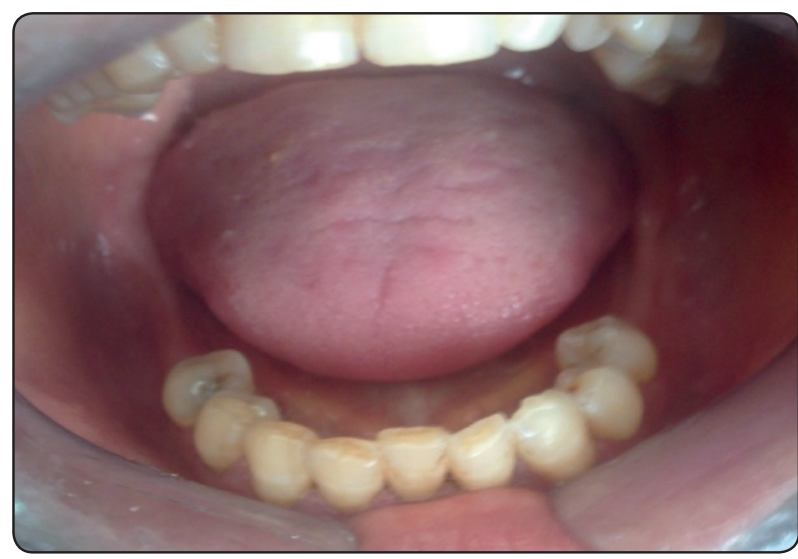

Fig (1) Intra-oral view, Kennedy class I lower partially edentulous ridge. 
selected and adjusted aluminum stock trays. The impressions were poured in dental stone* to produce study casts. Study cast mounted on a semi adjustable articulator $^{* *}$. A face bow ${ }^{* * *}$ record was used to mount the upper cast. A provisional interocclusal wax ${ }^{* * * *}$ record was made to mount the lower cast. A protrusive interocclusal wax record was made to adjust the articulator horizontal and lateral condylar guidance's. The occlusal plane was evaluated and over erupted teeth was identified. Slightly over erupted teeth were reduced and marked. The marked diagnostic casts were used as guide to perform the needed occlusal adjustments intra-orally. A cone beam $\mathrm{CT}$ (CBCT) radiograph was made to determine the bone height, width and the location of the inferior alveolar canal at the fixture site and to determine the length of the implant. Trial setting of artificial teeth was carried out. A transparent acrylic surgical stent was fabricated over the trial setting up. The acrylic stent rests anteriorly on the incisal edges of the remaining anterior teeth to provide guide for drills during implant insertion.

All patients receive implants with 3.75 diameters and $10 \mathrm{~mm}$ length were placed in the edentulous 1st molar area of the distal extension ridges using the standardized two-stage submerged surgical protocol. The retained partial overdenture design for all of the patients relied on lingual bar major connectors, bicuspid abutment with reverse Aker clasp assembly for retention-support, and indirect retention from canine cingulum rests. After construction of the mandibular cobalt-chromium alloy frameworks, an impression was recorded for the distal extension ridges using a medium body elastomeric(Impregum $\mathrm{F}$ and Permadyne LV; ESPE America Inc, Plymouth Meeting, Pa) and an altered cast impression technique was employed Semi-anatomic acrylic resin teeth (Vitapan ${ }^{\circledR}$,Vita Zahnfabrik, Bad Säckingen, Germany) were arranged to ensure balanced occlusal contact.

\footnotetext{
* LascodSpq, Sestofino(f1), Italy

** EasiCo,Ltd,Tokyo,Japan

*** Modeling Wax, Cavex, Holland
}

Patients were recalled after four months of fixture installation.

A panoramic radiograph was taken to confirm absence of any radiolucency around the implants. Fixture position was detected by palpatation and the surgical stent was used as a guide for implant position.

The implant site was detected by sterile explorer, local anesthesia was infiltrated at the implant site, and a sterile punch was used to expose the implant. The cover screw was unthreaded using hex screw driver. The selected patients were randomly divided into two equal groups, locator attachments were threaded to group 1 patients (fig 2) and ball and socket attachment to group 2 patients (fig 3) to provide retention for the prosthesis

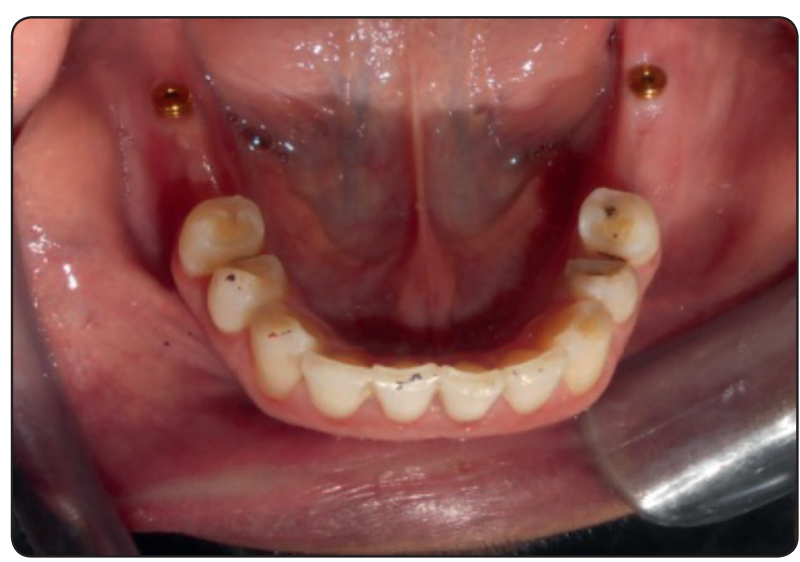

Fig. (2) Group 1 patients with locator attachments

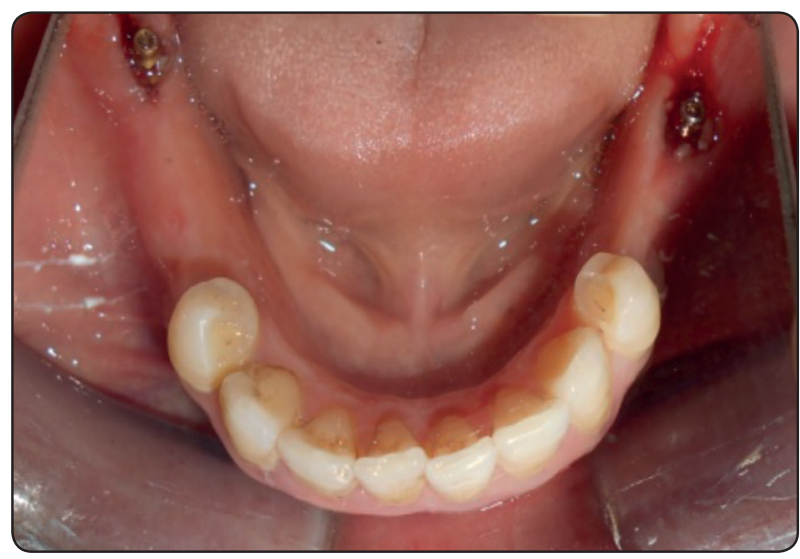

Fig. (3) Group 2 patients with ball and socket attachments 
Pick up technique was used to attach female housing of locator to the fitting surface of theprosthesis of the first group and the female housing of ball and socket to the second group patients.

Patients were frequently recalled for inspection and post insertion adjustments. Follow up visits were scheduled, six, and twelve months after denture insertion for making radiographic records evaluate the implant marginal bone height changes. Clinical evaluation was carried out to inspect the condition of denture bearing area, implant abutments, and oral hygiene Marginal bone height change around the implants was evaluated using the linear measurement system supplied by the cone beam computed tomography (CBCT) (K.V.P. 85, M.A. 16, Field of view $7 \times 14.5 \times 14.5 \mathrm{~cm})$.

Patients were instructed not to move during radiographic exposure. After exposure, the 3D image appeared on the computer screen display. The mesial, distal, buccal and lingual marginal bone height around the implants were evaluated, using the linear measurement system of the software (Ondemand 3D) with flat panel detector supplied by the cone beam $\mathrm{CT}$.

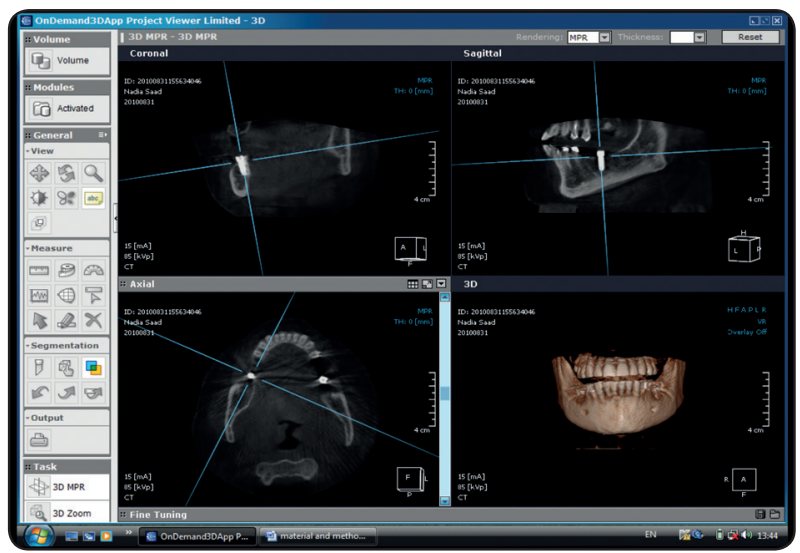

Fig (4): Different planes used to measure marginal bone height around abutments and implants.
From the sagittal plane, the distal and mesial marginal bone height around implants were evaluated. First a line was drawn horizontally tangential to the apex of the implant and perpendicular to its long axis. Two lines were then drawn tangential to the mesial and distal surfaces of the implant, parallel to each other and extending from the highest level of alveolar crest to the horizontal line. From the frontal plane, the buccal and lingual marginal bone height of the implants were evaluated following the same procedures (fig 4,5).

The measurements were carried out at the end of each follow up appointment (at insertion, six and twelve months post insertion). The marginal bone loss at different intervals was obtained by calculating the difference in bone height at that interval from the base line measurement.

\section{RESULTS}

This study was performed to evaluate bone height around implant with attachments during twelve months follow up period between ball/socket attachment and Locator ${ }^{\circledR}$ attachment.

Measurements were taken buccally, lingually, mesially and distally to the implant and mean and standard deviation were calculated for further statistical study.

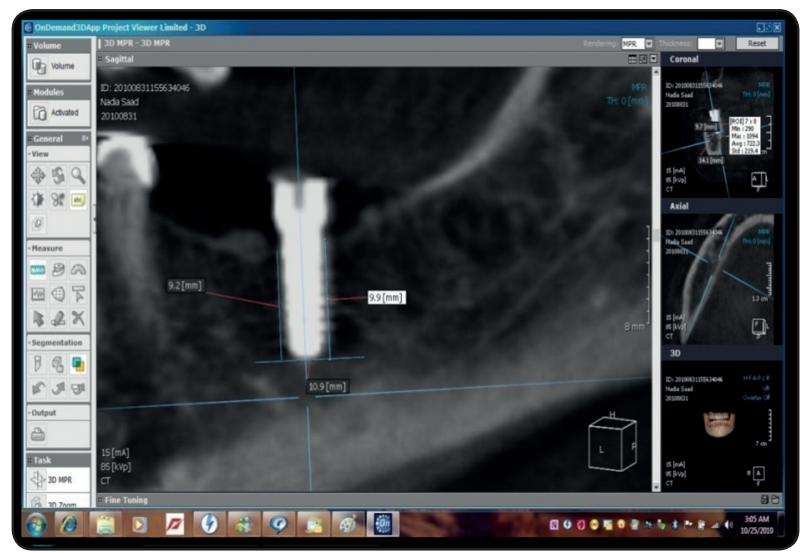

Fig (5): Mesial and distal marginal bone height around posterior implants were measured. (sagittal plane) 
For both groups individualy, one way analysis of variance (ANOVA) test was performed followed by Tukey`s post hoc test for multiple comparisons during twelve months follow up. It was revealed that there was significant difference between all follow up periods as $\mathrm{P}$-value $<0.05$, listed in table (1) and showed in figure (1).

For comparison between both groups for each follow up period, independent $t$ test was performed which revealed that there was insignificant difference between both groups for all follow up periods as P-value $>0.05$, listed in table (1) and showed in figure (1).

TABLE (1): Comparison between ball and locator attachment during twelve months follow up period:

\begin{tabular}{|c|c|c|c|c|c|}
\hline \multirow{2}{*}{$\begin{array}{c}\text { Bone } \\
\text { Height }\end{array}$} & \multicolumn{2}{|c|}{ Locator ${ }^{\circledR}$} & \multicolumn{2}{c|}{ ball/socket } & \multirow{2}{*}{ P-value } \\
\cline { 2 - 5 } & Mean & SD & Mean & SD & \\
\hline Baseline & $0.17^{\mathrm{a}}$ & 0.026 & $0.18^{\mathrm{a}}$ & 0.036 & $0.3905^{*}$ \\
\hline Six Months & $0.21^{\mathrm{a}}$ & 0.042 & $0.23^{\mathrm{b}}$ & 0.029 & $0.1403^{*}$ \\
\hline $\begin{array}{c}\text { Twelve } \\
\text { months }\end{array}$ & $0.32^{\mathrm{b}}$ & 0.041 & $0.35^{\mathrm{c}}$ & 0.063 & $0.1334^{*}$ \\
\hline P-value & \multicolumn{2}{|c|}{$0.00^{* *}$} & \multicolumn{2}{|c|}{$0.00^{* *}$} & \\
\hline
\end{tabular}

M; Mean, SD; Standard deviation

*insignificant difference * * significant difference

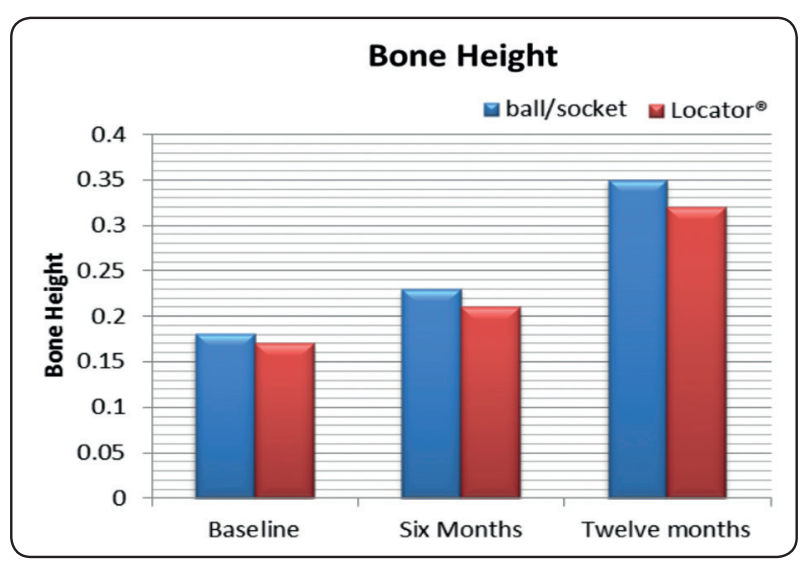

Fig. (6): Comparison between ball/socket and Locator $\AA$ duringtwelve months follow up period

\section{DISCUSSION}

The duality of support that characterizes distal extension removable partial dentures is often characterized by time-dependent adverse changes in both the abutment teeth and edentulous areas. Consequently, adjunctive implant support has been proposed for mandibular class I and II removable partial denture designs. The premise is that this will minimize the risk of potential problems of patient discomfort associated with prosthesis retention and stability resulting from residual ridge resorption. ${ }^{(13,14)}$.

Patients that participated in this study were carefully selected, examined and prepared to avoid any factor that may adversely affect the results of the study. Accurate patient selection and thorough clinical, laboratory and radiographic examinations were performed to exclude any condition that might affect the success of the dental implants or the results of the study Standard clinical and laboratory techniques were followed for denture construction for all patients to decrease variables that could affect the results of this study. ${ }^{(15,16)}$

In this study 2-stage implant technique was used to reduce risk of bacterial infection, prevent apical migration of epithelium along the body of the implant and decrease the risk of loading the implant early ${ }^{(17)}$

Implants were placed bilaterally at the distal extension of the denture base, in the area of the first molar area to minimize the denture displacement resulting from difference of support between abutments and residual alveolar ridge. The main objective of an implant located under the most posteriorly of the distal extension denture base is to stabilize the RPD in a vertical direction and provide maximum support and decrease the torquing action on the natural abutments anteriorly and preserve its crestal bone. Implants placed distally changed the Kennedy Class I tooth-tissue supported to that of the Class III tooth-implant supported ${ }^{(18,19)}$. 
CBCT digital imaging is as accurate as the digital imaging produced by conventional medical CT units and it is not affected by head posture as conventional CT. The distance of an anatomic structure (like the mandibular nerve) or thickness of cortical bone can be measured to one-tenth of a millimetre ${ }^{(20-22)}$.

Cone Beam CT (CBCT) offers a promising alternative approach since it provides sub-millimetre resolution images of high diagnostic quality, with short scanning time and reduced radiation dose up to 15 times lower than Multi-Slice CT scans $(\mathrm{MSCT})^{(23)}$.

Crestal bone resorption around the implants is a well-known phenomenon occurring mostly as an immediate bone response after implant insertion as well as after functional implant loading. Many studies ${ }^{(24-26)}$ confirmed these findings and agreed that most of this resorption occurs within the first year from loading. The amount of bone resorption occurring after loading may be related to many factors as the amount of load, nature of the prosthesis, bone quantity, quality and implant related factors. It is well known now in the literature that crestal bone resorption is not only unavoidable but also time related. A statistically significant difference between the two implant designs was reported for all the follow up periods.

Elimination many of the problems of distal extension cases were done by insertion of Implants in the second molar area to stabilize the removable partial denture in a vertical direction and effectively change the Kennedy class II into class IIII ${ }^{(27)}$.

The results of this study showed that bone changes with the locator was less than that occurred with ball and socket, both attachments provide freedom of movement in all directions. However the occluso gingival height of the locator is less than that of ball and socket. This could introduce less lateral stresses and consequently explain the difference in the bone changes around the implants ${ }^{(10,28)}$.
Within the limitations of this study, it is concluded that all the attachment systems were useful. No significant difference was observed between the attachment systems. Locator attachment was found more advantageous to ball;further studies are still needed, including the comparison of ball and Locator attachment systems used.

\section{REFRANCES}

1. Aurélio, M., Leonardo, R., Consani, X., Aurélio, M.,Antonio, S., \& Ponta, S. (2011). Association between implants and removable partial dentures : review of the literature. RSBO, 8(1), 88-92.

2. Alan B. Carr DMD MS, D. T. B. D. M. (2010). McCracken's Removable Partial Prosthodontics, 12th Edition.

3. Tandon, R., Gupta, S., \&Agarwal, S. K. (2010). Denture base materials: From past to future. Indian Journal of Dental Sciences, 2(2), $33-39$

4. Griffin, T. J.; Cheung, W. S.: The use of short, wide implants in posterior areas with reduced bone height: a retrospective investigation. The Journal of prosthetic dentistry 2004, 92, 139-44.

5. Shahmiri, R. A., \&Atieh, M. A. (2010). Mandibular Kennedy Class I implant-tooth-borne removable partial denture: A systematic review. Journal of Oral Rehabilitation, $37(3), 225-234$.

6. Gotfredsen, K.; Holm, B.: Implant-supported mandibular overdentures retained with ball or bar attachments: a randomized prospective 5-year study. The International journal of prosthodontics $2000,13,125-30$.

7. Trakas, T.; Michalakis, K.; Kang, K.; Hirayama, H.: Attachment systems for implant retained overdentures: a literature review. Implant dentistry 2006, 15, 24-34.

8. Alsiyabi, A. S.; Felton, D. A.; Cooper, L. F.: The role of abutment-attachment selection in resolving inadequate interarch distance: a clinical report. Journal of prosthodontics : official journal of the American College of Prosthodontists 2005, 14, 184-90.

9. Lee, C. K.; Agar, J. R.: Surgical and prosthetic planning for a two-implant-retained mandibular overdenture: a clinical report. The Journal of prosthetic dentistry 2006, 95, 102-5.

10. Evtimovska E, Masri R, Driscoll C F \& Romberg E. The Change in Retentive Values of Locator Attachments 
and Hader Clips over Time. Journal of Prosthodontics 18 (2009) 479-483

11. Zarb GA. \&Albrektsson T. Consensus report: Towards optimized treatment outcomes for dental implants. J Prosthet Dent .80: 641,1998.

12. Ma, S. \& Payne, A. G. Marginal bone loss with mandibular two-implant overdentures using different loading protocols: A systematic literature review. Int JProsthodont. 23: 117-126,2010.

13. Mitrani R, Brudvik JS, Phillips KM. Posterior implants for distal extension removable prostheses: A retrospective study. Int J Periodontics Restorative Dent 2003; 23:353359 .

14. Ohkubo C, Kobayashi M, Suzuki Y, Hosoi T. Effect of implant support on distal-extension removable partial dentures: in vivo assessment.Int J Oral Maxillofac Implants 2008; 23:1095-101.

15. Guler A, Sumer M, Sumer P and Bicer I : The evaluation of vertical heights of maxillary andmandibular bones and the location of anatomic landmarks in panoramic radiographs of edentulous patients for implant dentistry. J. Oral Rehabil, 32; 741, 2005.

16. Abuelross E.M, Shaarawy MA, and Shaker KT: The effect of varying partial denture design on the supporting structures of posterior implants used for bilateral distal-extension cases. Cairo dental journal, 21:87, 2005.

17. Chiapasco M, Abati S, Romeo E, Vogel G: Implant retained mandibular overdentures with Brammark system MKII implants : a prospective comparative study between delayed and immediate loading. Int J Oral and maxillofac Implants, 16,537, 2001.

18. Ohkubo C, Kurihara D, Shimpo H, et al: Effect of implant support on distal extension removable partial dentures: In vitro assessment. J Oral Rehabil 34:52, 2007.

19. Blum IG and McCord JF: A clinical investigation of the morphogical changes in the posterior mandible when implant retained overdentures are used. Clin Oral Implants Res, 15: 700, 2004.
20. Loubele M, Maes F, Schutyser F, Marchal G, Jacobs R and Suetens P: Assessment of bone segmentation quality of cone beam CT versus multislice spiral CT: a pilot study. Oral Surg Oral Med Oral Pathol Oral Radiol Endod, 102, $225,2006$.

21. Hashimoto K, Kawashima S, Araki M, Iwai K, Sawada K and Akiyama Y: Comparison of image performance between cone beam computed tomography for dental use and four-row multidetector helical CT. J Oral Sci, 48, 27, 2006

22. Ludlow J B, Laster WS, See M, Bailey LJ \& Hershey HG: Accuracy of measurements of mandibular anatomy in cone beam computed tomography images. Oral Surg Oral Med Oral Pathol Oral Radiol Endod, 103: 534, 2007.

23. Scarfe WC, Farman AG and Sukovic P: Clinical applications of cone-beam computed tomography in dental practice. J Can Dent Assoc, 72, 75, 2006.

24. Cocchetto, R.; Traini, T.; Caddeo, F.; Celletti, R.: Evaluation of hard tissue response around wider platformswitched implants. The International journal of periodontics \& restorative dentistry 30, 163-71,2010,.

25. Serrano-Sánchez P, C.-G. J., Manzanerapastore, LorrioCastro C, Bretones-López P, Pérezllanes JA.: The influence of platform switching in dental implants. A literature review. Med Oral Patol Oral Cir Bucal, 400-405,2011.

26. Enkling, N.; Johren, P.; Klimberg, T.; Mericske-Stern, R.; Jervoe-Storm, P. M.; Bayer, S.; Gulden, N.; Jepsen, S.: Open or submerged healing of implants with platform switching: a randomized, controlled clinical trial. Journal of clinical periodontology 38, 374-84, 2011.

27. Grossmann Y, Nissan J and Levin L: Clinical effectiveness of implant supported removable partial denture. A review of literature and retrospective case evaluation. J. Oral and Maxillofac. Surg67: 72. 2009.

28. Hong HR, Pae A, Kim Y, et al: Effect of implant position, angulation, and attachment height on peri-implant bone stress associated with mandibular two-implant overdentures: a finite element analysis. Int J OralMaxillofac Implants 27:e69-e76, 2012. 